\title{
Tuning GPCR-based sensors
}

\author{
The elimination of all cross-talk in a signaling pathway turns a yeast cell into \\ a model cell that can sense and report on metabolites.
}

B efore William Shaw joined the lab of Tom Ellis at Imperial College London as a $\mathrm{PhD}$ student, his interest in G-protein-coupled receptor (GPCR) signaling was kindled during an internship at AstraZeneca. When the biopharmaceutical company suggested a joint project to engineer yeast cells as senseand-response systems for medically relevant markers, Ellis liked the idea of combining his group's expertise in genome engineering and synthetic biology with the knowledge of GPCR signaling from the company.

The first challenge the researchers faced was that though yeast is a relatively simple organism, its signaling pathways are anything but. Ligand binding to a GPCR triggers a signaling cascade with a lot of cross-talk and feedback loops before arriving at the expression of a target gene.

Having so many players makes the tuning of output based on input difficult. In preliminary experiments, Shaw found that it was not trivial to correlate a ligand concentration with a desired amount of output. While a lot of work has been done on GPCR signaling in yeast over the past 15 years, Ellis and his team realized that no one had tackled the problem of how to create a simple pathway that is not interconnected with many other pathways so that a clear line from input to output can be established.

Ellis teamed up with a group at Cambridge led by Graham Ladds, who brought expertise in mathematical modeling of signaling cascades and could identify the essential components of a signaling cascade.

Back in the lab, Shaw focused on the pheromone-response pathway, a nonessential signaling cascade for yeast, which can reproduce asexually and maintains the pheromone system only, as Ellis puts it, "in the hope that one day they will have sex." Shaw used CRISPR to make up to 15 deletions in the yeast genome to re-create the streamlined model Ladds had developed. Ellis described the result: "it was now a narrow pathway that was not interacting with anything else."

Thus simplified, the cascade was ready for fine-tuning. To Ellis's surprise, the signaling cascade was very sensitive to the relative amounts of the $\alpha$-, $\beta$-, and $\gamma$-subunits that make up the trimeric $G$ proteins, which are the first proteins to conduct the signal from the GPCR to downstream effectors. "Quite small differences in expression of one over the other will change the leakiness from no leakiness to being accidentally on half of the time," explains Ellis.

The team also optimized the amount of GPCR and created a synthetic transcription factor at the end of the cascade that controlled the expression of a reporter gene. By tuning the different components, the researchers could increase or decrease the response range depending on the application needed. For example, if a linear signal is desired-a proportional increase between input and output signal-the range of operation should be large; if a digital yes-or-no answer is needed to detect the presence or absence of a metabolite, the range should be narrow.

In an attempt to make the system more sensitive than its wild-type counterpart, Shaw developed a two-cell system. The first strain, called the amplifier cells, recognized a ligand of interest and in response produced the $\alpha$-factor pheromone, which then triggered expression in the reporter cells. This system allowed the team to detect a pathogenic peptide at 1,000-fold increased sensitivity compared with that of previous approaches.

Ellis sees more applications for this system in yeast. As an example, he cites sensing of cannabidiol (CBD), a nonpsychoactive compound in marijuana that Jay Keasling's group at UC Berkeley is synthesizing in yeast. "The yeast will tell you which CBDs are being made at which concentrations," he says.

In addition, he is heading a project aimed at establishing yeast cells that work in coordination and mimic the multicellular behavior of tissues. "We want to use yeast to help us understand how these things have naturally evolved," Ellis describes.

And yeast is not the end point; Ellis concludes, "We would like to re-create this system in mammalian cells, particularly in cells that circulate in the blood."

\section{Nicole Rusk}

Published online: 30 May 2019 https://doi.org/10.1038/s41592-019-0440-9

Research papers

Shaw, W. M. et al. Engineering a model cell for rational tuning of GPCR signaling. Cell 177, 782-796 (2019).

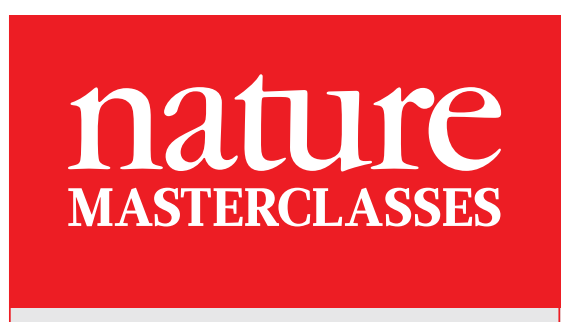

\section{Online Course in Scientific Writing and Publishing}

Delivered by Nature Research journal editors, researchers gain an unparalleled insight into how to publish.

Try a free sample of the course at
masterclasses.nature.com
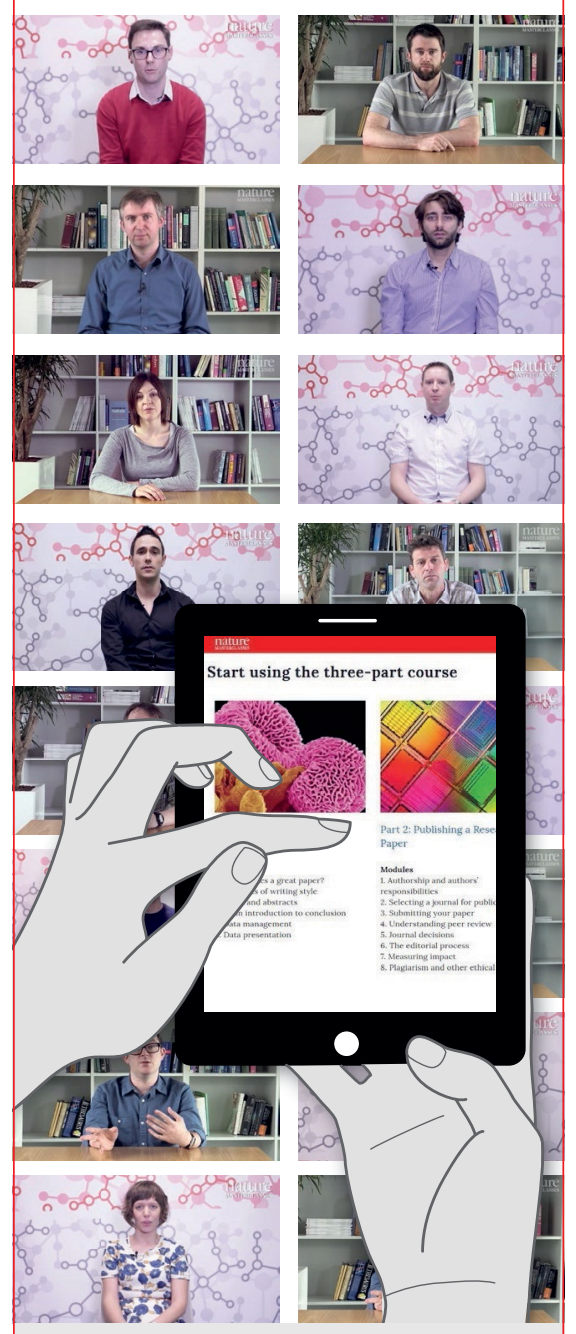

Bite-size design for busy researchers Subscribe as a lab or institution

W masterclasses.nature.com in Follow us on Linkedln

f Skills and Careers Forum for Researchers 